\title{
Inconscient et refoulement selon la phénoménologie de la vie
}

\author{
Nuno Miguel PROENÇA \\ Université Nouvelle de Lisbonne
}

Dans un ensemble considérable de travaux, dont le plus important est sans doute la Généalogie de la Psychanalyse, Michel Henry interroge le statut de la psychanalyse de façon originale et parfois même polémique. Sans jamais évoquer la relation entre Freud et le freudisme ce qu'il affirme de la relation entre Marx et le marxisme, Henry ne cesse de mettre en évidence la façon dont une certaine lecture de la théorie freudienne, dans sa tentation idéologique - et dans les excès auxquels elle peut mener - cache ce que la pensée de Freud recèle d'original. Par ailleurs, l'auteur de la Phénoménologie matérielle affirme tenter d'esquisser une nouvelle interprétation de la relation qui existe entre phénoménologie et psychanalyse, dans la mesure où il «propose une nouvelle conception de la phénoménologie " ${ }^{1}$ et attire aussi notre attention sur le fait que, contrairement aux sciences humaines, la psychanalyse « résiste à la réduction galiléenne ${ }^{2}$ en cela qu'elle " affirme et maintient, fût-ce sans le savoir, le droit invincible de la vie ${ }^{3}$ dans ce que celle-ci a d'irrémédiablement subjectif, alors que l'humanité est ravagée par le savoir objectiviste et par ses prétentions exorbitantes. Selon Henry, la psychanalyse reconnaît, en effet, "l'immanence radicale de la subjectivité absolue dans son hétérogénéité ontologique au monde de la représentation » ${ }^{4}$, tout en conservant le présupposé scientiste selon lequel seul existe ce qui est objectif et déterminable objectivement : «l'inconscient psychique, reconnu finalement comme l'affect, n'est encore que le représentant de processus bio-énergétiques, donc d'une réalité naturelle $»^{5}$, ce qui va donner naissance à une tension au sein de la théorie qui s'exprimera aussi au niveau de la pratique clinique et des formes de connaissance (de soi) que celle-ci rend possibles.

En situant dans l'historial de la métaphysique occidentale quelques-unes des notions clés de la théorie freudienne, Henry nous montre, à la lumière de sa

\footnotetext{
${ }^{1}$ Michel Henry, « Phénoménologie et psychanalyse », dans Psychiatrie et existence, P. Fédida et J. Schotte (éd.), Grenoble, Jerôme Million (coll. « Krisis »), [1991] 2007, p. 95.

${ }^{2}$ Michel Henry, B, p. 163.

${ }^{3}$ Ibid. Henry écrit aussi, dans l'introduction de sa Généalogie de la Psychanalyse, que "[1]a psychanalyse n'appartient donc pas au corps des sciences humaines auquel on la rattache aujourd'hui et dont elle sera ici soigneusement dissociée : elle en est bien plutôt l'antithèse » (Michel Henry, GP, p. 12).

${ }^{4}$ Michel Henry, B, p. 162.

${ }^{5}$ Ibid.
} 
phénoménologie de la vie, que le médecin de Vienne est un héritier des élaborations philosophiques qui l'ont précédé. Sa compréhension de la psyché est en partie une conséquence de la réduction de l'essence originelle de la phénoménalité à la représentation, telle qu'elle a été produite par le cogito cartésien. Mais elle est aussi une continuation de ce que la réflexion schopenhauerienne et nietzschéenne nous donnent à entendre de la vie irréductible à la phénoménalité dont elle est formatrice, et c'est à ce titre que Michel Henry reconnaît à la théorie freudienne " une portée ontologique immense $»^{6}$. Elle affirme, en effet, que le «fond de la psyché » échappe à jamais à la pensée et à son Ek-stase représentative, qu'il se situe en deçà de celle-ci et la conditionne. À ce Fond, la psychanalyse donne le nom d'inconscient.

Or, dans la métapsychologie freudienne, le concept d'inconscient est indissociable de celui de refoulement. C'est à cause de ce à quoi Freud donne le nom de « refoulement » que le centre de la psyché est déplacé, et qu'il n'est plus identifiable à la conscience et à ses représentations. C'est en raison du refoulement que l'inconscient est formé comme zone inaccessible à celles-là et, régie par un processus singulier (le processus primaire) à l'œuvre de façon manifeste dans le travail du rêve, du lapsus, du mot d'esprit et dans la formation des symptômes ; c'est dans cette même zone qui est maintenue à l'écart de la conscience par un effort constant (le refoulement) que se trouve, selon Freud, le centre de la vie psychique. Dans la Généalogie de la Psychanalyse, la lecture de l'inconscient qui est effectuée par Michel Henry semble radicaliser ce déplacement. Mais si Henry s'appuie sur l'hypothèse freudienne selon laquelle c'est parce qu'on ne peut se fuir soi-même que le refoulement a lieu, l'originalité de sa lecture attire notre attention sur le fait que la force inhérente au refoulement est d'ordre affectif et qu'elle est formatrice des représentations, et n'est pas seulement un effort pour les maintenir à distance. L'énergie à l'œuvre dans le refoulement est celle de la vie elle-même, par rapport à laquelle il n'y a pas d'écart possible : l'inconscient, plutôt qu'une zone de représentations séparée de la conscience, se confondrait avec l'activité incessante de la vie, au point de ne pas exister.

Tout au long de cet article, nous nous proposons d'identifier, en premier lieu, les arguments sur lesquels se fonde cette lecture qui relie de façon originale inconscient et refoulement, en commençant par repérer le rapport entre le décentrement de la conscience, chez Freud, et les conséquences que Michel Henry en retire, notamment pour ce qui concerne les rapports entre ipséité et passibilité. Ensuite, nous poursuivrons en identifiant, avec Henry, un moment essentiel de la généalogie philosophique de la notion de refoulement et la façon dont il noue mémoire, perception et raison (ou folie) à une dimension de la vie qui est irréductible à la représentation consciente. Enfin, après avoir évalué les conséquences que cela peut

${ }^{6}$ Michel Henry, GP, p. 8.

${ }^{7}$ Ibid. 
provoquer quant à la détermination de la signification du concept d'inconscient pour la phénoménologie de la vie, nous esquisserons alors les lignes de fond de sa critique de la psychanalyse.

\section{Pulsions, refoulement et inconscient}

Dans la métapsychologie freudienne, le décentrement de la conscience - et son conditionnement par des processus qui ne relèvent pas de son initiative - n'est à l'origine de la constitution d'une zone psychique inconsciente que si les représentants de la vie organique au sein de la vie psychique font l'objet d'un refoulement. C'est par le biais de cette notion que Freud rend compte d'un jeu de forces en raison duquel est constituée une zone de la vie psychique composée par cela qui, non seulement n'est pas en mesure d'accéder à la conscience, mais ne cesse pas - pour autant - de produire des effets sur le cours de la vie consciente et de ses représentations. La formation, au sein de la vie psychique, d'une zone inconsciente destitue le moi du rôle central qu'il s'arrogeait au sein de la vie psychique et inflige à l'humanité, dans les mots de Freud, la dernière d'une série de blessures narcissiques qui doivent l'inviter à l'humilité. Les deux premières ont été les conséquences des découvertes de Copernic et de Darwin: non seulement l'humanité, en habitant la planète Terre, n'est pas au centre de l'univers, mais en plus, elle n'advient qu'après un processus d'évolution des espèces vivantes. Enfin, la psychanalyse montre que le Moi n'est pas maître dans sa propre maison, et que c'est l'inconscient qui y règne. Non seulement les processus psychiques ne deviennent accessibles et subordonnés au moi que par une perception incomplète et incertaine, mais notre vie pulsionnelle révèle encore une passivité de fond en raison de laquelle, en nous apparaissant, nous nous apparaissons toujours et déjà impliqués dans un déjà-là d'une vie dont nous ne sommes pas à l'origine et qui nous situe dans un méandre de sensations et de besoins, de significations et de désirs, d'interdits, de règles et d'usages qui ne relèvent pas de notre initiative et qui conditionnent notre vie vigile et intentionnelle. Ce ne sont pas seulement la faim, la soif, les besoins qui résultent de l'ingestion et de la digestion d'aliments, la respiration ou autres efforts d'auto-conservation issus d'une activité incessante et pulsionnelle qui placent la vie consciente après le dynamisme organique d'un corps vivant et vécu. Ce sont aussi le plaisir et le désir sexués. Or, selon Freud, ce sont précisément les pensées, les images ou les souvenirs liés aux pulsions sexuelles qui font le plus souvent l'objet d'un refoulement qui donne lieu à la formation d'une zone psychique inconsciente et qui déplace le centre de la vie psychique vers une zone autre que celle du Moi. 
Freud nous présente le refoulement comme « la pierre angulaire de l'édifice de la psychanalyse ${ }^{8}$ et le définit comme un des quatre destins possibles des pulsions. Dans le lexique freudien, la Trieb apparaît définie par les notions de "source », «but », « objet » et « poussée » ${ }^{9}$. Elle est un processus dynamique, qui consiste dans une charge énergétique, elle-même facteur de motricité ; c’est cette charge que Freud nomme « poussée » et en raison de laquelle l’organisme tend vers un «but ». La «source » de la pulsion est une excitation corporelle, qui est l'état de tension faisant pression et son "but " consiste à supprimer la tension qui se situe à sa source. La pulsion atteint son but grâce à son « objet ». C’est en lui, ou par lui, que la tension issue de la source et, créant une pression, est supprimée : c’est là ce que l'on identifie comme étant le plaisir, ou la satisfaction, et qui constitue le véritable but de la pulsion.

À la différence des excitations qui viennent de l'extérieur, qui ont un impact unique et qui peuvent être supprimées par l'emploi de leur charge énergétique dans un type d'action approprié, comme la fuite, les excitations pulsionnelles ont une force d'impact permanent, qui est indice du monde intérieur. Si les excitations, dont la physiologie a donné le concept, sont rattachées au schéma du réflexe - selon lequel l'excitation qui est apportée de l'extérieur au tissu vivant est également déchargée vers l'extérieur sous la forme d'une action unique appropriée, qui soustrait la substance excitée à l'effet de l'excitation et l'éloigne de son champ d'influence - l'excitation pulsionnelle, en revanche, en venant de l'intérieur de l'organisme, agit de manière différente sur le psychique et exige d'autres actions qui seront seules capables de les éliminer. Elle « attaque de l'intérieur » ${ }^{10}$ du corps. Sa force d'impact est "constante " ${ }^{11}$, et jamais "momentanée " ${ }^{12}$. Ses destins essentiels, puisqu'on ne peut en supprimer la source (ce qui reviendrait à se supprimer). Il y en a donc quatre : l'inversion dans leur contraire, le directionnement sur la personne propre, le refoulement ou la sublimation. Mais en aucun cas la fuite.

Selon Freud, le troisième de ces destins, le refoulement, est une négation spécifique des pulsions, qui ne les détruit pas, mais qui les suspend. Son essence consiste ainsi à " écarter et maintenir à distance du conscient ${ }^{13}$ les représentants des pulsions et à les circonscrire dans cette zone de la vie psychique nommée inconscient. Dans les textes métapsychologiques de Freud, un tel écart a lieu pour des raisons affectives. Sont refoulés les représentants des pulsions dont la satisfaction provoquerait du déplaisir ; ou plutôt (et parce que Freud présume que le

\footnotetext{
${ }^{8}$ Sigmund Freud, «Contribution à l'histoire du mouvement psychanalytique », dans Essais de Psychanalyse, Paris, Payot, 1936, p. 273.

${ }^{9}$ Sigmund Freud, «Pulsion et destins des pulsions », dans Métapsychologie, trad. par J. Laplanche et J.-B. Pontalis, Paris, Gallimard (coll. « Folio »), p. 18-19.

${ }^{10}$ Ibid., p. 14

${ }^{11}$ Ibid.

${ }^{12}$ Ibid.

${ }^{13}$ Sigmund Freud, « Le refoulement », dans Métapsychologie, op. cit., p. 47.
} 
but de la pulsion est la satisfaction) ; sont refoulées celles dont la satisfaction provoquerait simultanément plaisir et déplaisir, ce dernier étant en quantité supérieure. Quelle que soit son intensité néanmoins, la force d’inhibition provoquée par le refoulement ne peut entièrement détruire ni la pression pulsionnelle, ni sa source (ce qui équivaudrait à supprimer l'organisme vivant). Son type de refus particulier ne détruit pas non plus ses représentants demeurés inconscients sous forme de représentations.

Ces arguments s'avèrent essentiels pour Michel Henry, qui va en saisir la signification pour la caractérisation de la subjectivité, de l'ipséité et aussi du rapport entre refoulement et inconscient à la lumière d'une phénoménologie de la vie. Dans le cas des pulsions, nous dit Freud, « la fuite ne sert à rien, parce que le moi ne peut se fuir lui-même ${ }^{14}$. Même si la pulsion peut ne pas avoir une représentation mentale (Vorstellung) qui en est le lieu tenant (Repräsentant) au niveau de la vie consciente, elle ne cesse de se manifester par l'épreuve de son représentant affectif, qui jamais ne devient inconscient. Henry y signale la passibilité foncière de l’ipséité : autrement dit,

la constance de l'affection et le fait qu'il ne soit pas possible de lui échapper pour la fuir, de développer un écart, une différence, de prendre le moindre recul à son égard, c'est-à-dire à l'égard de soi-même ne qualifie rien d'autre que la subjectivité absolue et ainsi, en tant qu'affection immanente de soi par soi, l'essence de l'ipséité, et par conséquent, de cette façon, le moi (non plus nommé de l'extérieur, mais impliqué dans sa passibilité la plus intérieure et la plus inaliénable). ${ }^{15}$

Prise en soi-même et prisonnière de soi-même, une telle affection - bien qu'étant le résultat d'une poussée relevant d'une altérité de la pulsion - est auto-affection et la subjectivité absolue se confond avec l'activité incessante dont elle n'a pas l'initiative. La force interne et formatrice de la vie se révèle ainsi au vivant, dont le corps est le lieu. C'est là qu'a lieu l'auto-révélation affective de la vie en tant qu'elle est vécue par un vivant toujours déjà impliqué dans une communauté. Par ailleurs et pour cette raison, la perception elle-même serait travaillée du dedans par ces strates de réalité pré-intentionelle et passive, à la fois pulsionnelles et affectives - qui l'informeraient - de façon à ce que la matière de la donation, à partir de laquelle se composent les formes perçues, aurait une force formatrice inhérente à l'activité du sujet percevant. Si le poids de la vie qui s'auto-affecte se fait trop lourd, c'est-à-dire, s'il y a souffrance, la vie s'efforce alors d'échapper à soi, de se fuir soimême. En raison du lien qui la lie à elle-même et qui ne peut être brisé, cela n’est pas possible et mène à un effort de changement. Ainsi Henry reprend-il « de

\footnotetext{
${ }^{14}$ Ibid., p. 45.

${ }^{15}$ Michel Henry, GP, p. 373.
} 
l'intérieur » la description freudienne de l'excitation pulsionnelle. On retrouvera cette lecture dans l'article « Psychanalyse et phénoménologie », publié en 1991 :

Cet effort de la vie pour modifier sa tonalité et pour se modifier soi-même c'est la pulsion. Et voilà comment la vie dans son affectivité et en raison de celle-ci devient cette tension intérieure inlassable elle aussi par laquelle elle s'efforce sans cesse de se transformer soi-même, très précisément de changer le malaise qui lui vient de sa propre subjectivité en autre chose de plus supportable comment l'Affect se fait Force. ${ }^{16}$

\section{Refoulement, volonté et représentations}

Le rapport particulier entre la Force, la Forme et l'affectivité, décelé ici par le biais d'une réflexion sur l'essence du refoulement et retrouvé dans les descriptions phénoménologiques de la pulsion, dont nous ne donnons ici qu'un exemple, permet à Henry d'identifier un des héritages métaphysiques de Freud. Il s'agit d'une ontologie nouvelle, celle de Schopenhauer qui, d'après l'auteur de la Généalogie de la Psychanalyse, «découvre l'affectivité comme révélation de l'Etre en lui-même, comme la matière dont il est fait, comme sa substance et sa chair » ${ }^{17}$. Freud s'inscrit donc dans cette lignée selon laquelle la manifestation phénoménale ne saurait se réduire à la représentation, d'autant plus que c'est le concept de refoulement luimême, comme " processus psychique par lequel certains faits eux-mêmes psychiques en repoussent d'autres, leur interdisant, sinon l'existence, du moins l'accès à la conscience ${ }^{18}{ }^{18}$ qui a été introduit pour la première fois dans la pensée occidentale par Schopenhauer. Au dire de Freud lui-même, nous rappelle Henry, la notion schopenhauerienne de volonté «équivaut aux [pulsions] de la psychanalyse ${ }^{19}$. Le rapport qu'il existe entre volonté et représentation chez Schopenhauer est analogue à celui qui existe entre pulsions et représentations en psychanalyse. Cette Volonté qui ne peut pas ne pas vouloir est semblable aux désirs qui laminent notre corps, ou plutôt identique à eux, elle est cette activité toujours recommencée, qui nous traverse, que nous sommes et à laquelle il ne nous est pas possible d'échapper. Le rapport qu'elle entretient avec l'affectivité et leur situation respective dans leur rapport avec l'essence de la vie se décide, nous dit Henry, dans « l'analyse extraordinaire où se trouve reconnu pour la première fois et cerné dans sa possibilité le phénomène du refoulement $»^{20}$.

On trouve ainsi ce concept de refoulement à propos de trois problèmes auxquels Schopenhauer allait «apporter des solutions absolument originales » : celui de la

\footnotetext{
${ }^{16}$ Michel Henry, « Phénoménologie et psychanalyse », op. cit, p. 102.

${ }^{17}$ Michel Henry, GP, p. 10.

${ }^{18}$ Michel Henry, « La question du refoulement chez Schopenhauer », dans PV-II, p. 131.

${ }^{19}$ Ibid., p. 133.

${ }^{20}$ Michel Henry, GP, p. 225.
} 
mémoire, celui de la perception et celui du rapport entre raison et folie $»^{21}$. Reprenons-les un à un.

Selon Henry, la théorie schopenhauerienne de la mémoire est une théorie du refoulement, car elle se situe dans la caractérisation générale du rapport entre volonté et représentation. Entendue selon la doctrine de Schopenhauer, une représentation est un double irréel de la force qu'est la volonté. Si la première est entièrement "passive ${ }^{22}$, la seconde est 《 toute-puissante ${ }^{23}$; si la première est «lumière $~^{24}$ et "conscience ${ }^{25}$, mais privée de force, la seconde est la force, mais elle est «privée de la représentation et de sa lumière ${ }^{26}$, de telle façon que ces deux instances se définissent par leur "exclusion réciproque ${ }^{27}$. C'est parce que, dans son analyse, «la volonté est sans représentation et la représentation sans volonté ${ }^{28}$ que la théorie schopenhauerienne du psychisme peut être dite "une théorie du refoulement $»^{29}$ qui a déjà en commun avec la théorie de Freud le fait que la vie de la conscience est déterminée par cela qui n'a pas de lumière, c'est-à-dire, "par le pouvoir lui-même aveugle de nos instincts et de nos pulsions ${ }^{30}$. Si l'essence de celui-ci est définie comme ne consistant que dans le fait d'écarter et de maintenir à distance du conscient, nous la retrouvons chez Schopenhauer lorsqu'il nous signale que :

la formation de la représentation lors du rappel du souvenir ou de son rejet [quand le souvenir est refoulé] s'explique par la Volonté. Telle ou telle représentation particulière est ou n'est pas voulue et, dans ce dernier cas, c'est la volonté qui « la tient en quelque sorte couverte de la main ${ }^{31}$

La représentation ainsi recouverte demeure bien, mais elle n'est pas admise dans la conscience. Son rejet équivaut à la forme particulière de négation du refoulement, qui ne détruit pas, mais conserve, et qui s'accompagne d'un savoir. Henry nous rappelle que ce processus est exactement celui que Freud a décrit dès 1893 dans les Études sur l'hystérie : il s'agissait de choses que le malade voulait oublier et qu'intentionnellement il maintenait, repoussait hors de sa pensée consciente. La mémoire, chez Schopenhauer, mais aussi chez Freud, n’est une faculté de la représentation qu'en apparence. Le contenu représenté est conduit devant l'esprit, non pas par une capacité de le produire en tant qu'objet, «c'est le pouvoir, en soi

\footnotetext{
${ }^{21}$ Michel Henry, « La question du refoulement chez Schopenhauer », dans PV-II, p. 131.

${ }^{22}$ Ibid., p. 134.

${ }^{23}$ Ibid.

${ }^{24}$ Ibid.

${ }^{25}$ Ibid.

${ }^{26}$ Ibid.

${ }^{27}$ Ibid.

${ }^{28}$ Ibid.

${ }^{29}$ Ibid.

${ }^{30}$ Ibid., p. 135.

${ }^{31}$ Ibid., p. 136.
} 
étranger, à cette production, qui lui permet de s'accomplir ou qui le lui interdit $»^{32}$. Cela se traduit, chez Schopenhauer comme chez Freud, par une rupture du fil de la mémoire, par une brisure de son enchaînement continu. L'absence qui vient rompre et briser le fil de la mémoire et la chaîne des représentations est un pouvoir autre, qui interdit la représentation. Il s'agit de la volonté, en tant qu'elle est à la fois la condition de la mémoire et de l'oubli : « ne pas vouloir que tel contenu entre dans la lumière de la représentation relève du même principe que celui qui soutient la mémoire, en est la simple détermination négative. $»^{33}$

C'est donc bien le refoulement qui se trouve ainsi défini par Schopenhauer, avec cette exclusion par la volonté d'une représentation hors du domaine de la conscience. Dans une telle présentation du lien généalogique qui existe entre le concept de refoulement tel qu'il se dessine chez Schopenhauer et celui qui est développé par Freud, nous apprenons également que la dichotomie entre le vouloirvivre et les représentations est constitutive de la Psyché. Le fait que l'instinct aveugle détermine la conscience représentative est "la loi générale ${ }^{34} \mathrm{du}$ psychisme. Il s'agit, nous dit Henry, de «l'expression permanente et incontournable de sa structure essentielle ${ }^{35}$, de celle-là qui, en termes freudiens, conduira à une subordination du Moi aux forces inconscientes en raison desquelles il ne sera plus maître de sa maison. Autrement dit, le décentrement provoqué par le refoulement se trouve déjà chez Schopenhauer, de même que la « manipulation de la représentation par la pulsion ${ }^{36}$ attestée par toute vie imaginaire et en raison de laquelle l'association d'idées est utilisée par la psychanalyse pour tenter de dévoiler l'inconscient. C'est parce que le rapport de condition à conditionné qui existe entre le Vouloir et la représentation se retrouve chez Freud comme rapport entre inconscient et conscient que le travail clinique se proposera comme tâche d'induire le second à partir du premier précisément en levant les refoulements. Or, se demande Henry, pourquoi la volonté (comme le désir ou la pulsion) refoule-t-elle la représentation? " Non pas à cause de son contenu représentatif mais de son affectivité ${ }^{37}$ et, « si le refoulement appartient à la constitution fondamentale de la psyché comme une de ses lois les plus constantes et les plus profondes, c'est d'abord parce que la représentation qu'il s'agit de refouler - ou d'accueillir - est déterminée affectivement [;] c'est en raison de cette affectivité seulement qu'elle est l'objet d'un rejet et que celui-ci peut et doit se produire ${ }^{38}$.

Il en va de la perception comme de la mémoire et de l'oubli : c'est encore la Volonté qui voudra ou ne voudra pas. Certains fragments du champ perceptif seront

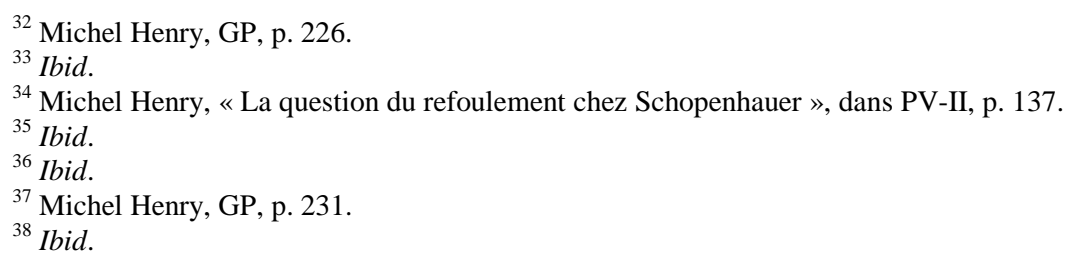


ou ne seront pas voulus. L'ouverture et l'anticipation perceptives seront façonnées par la force formelle de la volonté en tant que celle-ci portera ou détournera le regard en « rejetant alors un objet ou une partie de cet objet hors représentation et ainsi hors conscience, et le maintenant, à distance du conscient, comme le dit Freud $»^{39}$ en raison de ce qu'il suscite. Autrement dit, c'est parce qu'une représentation est affectivement déterminée ou investie d'affect qu'il peut y avoir un refus de son entrée dans l'ensemble de celles qui composent la vie consciente, et non à cause de son contenu. Michel Henry souligne, à ce propos, les expressions de Schopenhauer qui font référence au chagrin causé par une pensée, à la cruauté d'un souvenir qui devient pour cela insupportable, à l'angoisse qu'il suscite, ou à la répugnance provoquée par les représentations qui nous blessent. Lorsque les forces d’un individu sont dépassées, la nature recourt en conséquence à la folie.

La théorie de la folie rend compte du même clivage dans le psychisme de la force pulsionnelle et de la représentation. Elle préfigure de façon saisissante, ici encore, nous dit Henry, les conceptions freudiennes. L'origine de la folie, c'est justement le refus de la volonté de laisser pénétrer dans la conscience une représentation qui lui est contraire en raison d'une répugnance ou d'un excès. Alors que l'expérience dite «normale » est conçue par Schopenhauer comme un processus douloureux et une impression pénible par lesquels (et bien qu'étant contraires à la volonté) des représentations - qui constituent ensemble et dans leur liaison nécessaire l'ordre du monde - doivent parvenir à la conscience et être reconnues comme telles, le refus de la volonté de laisser entrer dans l'esprit une représentation déplaisante et de la rejeter hors de la conscience provoquera un trou qui sera à l'origine de la folie. Il s'ensuit que, dans l'hypothèse d'une représentation ainsi refoulée, «le tissu des représentations qui forme le monde présente brusquement une lacune, un vide laissé par cette représentation absente ${ }^{40}$, qu'il faudra combler par une autre qui n'est pas à sa place ou qui sera inventée à cette fin et qui défera, en raison de ce déplacement, l'ensemble cohérent de représentations dans lequel consiste le monde. Cela sera le cas, qu'il s'agisse de celles qui définissent le présent ou de l'ensemble des représentations passées ou à venir - lesquelles devront, elles aussi, se soumettre à certaines règles sans lesquelles elles ne seraient pas possibles. Ce qui est produit est une liaison arbitraire de phénomènes, un passé arrangé en cela que le contenu des souvenirs est falsifié et le fil associatif qui les lie est rompu. Que ce soit en termes de mémoire ou d'anticipation, le naturant de la projection représentative est la volonté. Les représentations sont (ou ne sont pas) repoussées en raison de leur tonalité, en raison de ce qu'elles ont de désagréable ou d'intolérable, mais il faut aussi que le vouloir soit lui-même originellement constitué en son être comme affectif, qu'il ne soit pas pur : « Ce n'est [...] jamais la volonté comme telle, un

\footnotetext{
${ }^{39}$ Michel Henry, « La question du refoulement chez Schopenhauer », dans PV-II, p. 136.

${ }^{40}$ Ibid., p. 136.
} 
vouloir pur, qui écarte la représentation mais seulement un vouloir préalablement déterminé en soi comme affectif, comme répugnance, blessure, dégoût, honte. ${ }^{41}$

Toutefois, si la doctrine de Schopenhauer anticipe la théorie freudienne du refoulement, elle en recèle aussi les apories. Henry les met en évidence en constatant que pour permettre la venue à l'esprit d'une représentation ou pour l'interdire, il faut d'abord savoir ce qu'est une représentation et la connaître de quelque façon, ne serait-ce que pour apprécier sa convenance ou sa non convenance au désir. Mais, si elle qui ne connaît rien et qui se définit par l'absence de connaissances (Erkenntnislosigkeit), comment la volonté pourrait-elle bien, sans représentation, posséder ou acquérir une telle connaissance nécessaire à rejeter une représentation ?

Cette aporie qui se trouve au cœur de la théorie du refoulement dès sa première formulation chez Schopenhauer atteint aussi toute conception qui voudra expliquer comment un pouvoir dépourvu de conscience peut bien écarter une représentation qu'il ne connaît d'aucune façon. C'est dire qu'elle se retrouve en toute philosophie faisant de l'inconscient le principe organisateur du psychisme et à plus forte raison, chez Freud et en psychanalyse. Mais elle ne subsiste que si nous restreignons le savoir à la conscience représentative. Si nous acceptons qu'il y ait des formes de manifestation de ce savoir qui ne requièrent pas la représentation, l'aporie peut se résoudre. Henry nous indique cette solution en signalant que le savoir inhérent à la volonté, celui qui se trouve à l'œuvre dans la formation ou la non-formation des représentations, est affectif. C'est cette nouvelle théorie - proprement henryenne qui rend intelligible le déplacement essentiel - entrevu par Schopenhauer mais non porté par lui à la clarté du concept - en vertu duquel toutes les facultés représentatives, à savoir l'œil, la mémoire, la pensée, trouvent désormais leur principe dans un pouvoir qui n'est plus celui de la conscience intentionnelle : dans la vie. Or, celle-ci est auto-affection. C'est là que se trouverait, paradoxalement le centre de l'inconscient : dans une auto-affection qui jamais ne devient inconsciente, mais qui n'est pas non plus à confondre avec la conscience, définie par la représentation. En sachant cela, et en suivant la réflexion de Michel Henry, nous avons désormais la possibilité de lire philosophiquement Freud :

en tant que l'acte proto-fondateur de l'extériorité qui sert de fondement à toute représentation possible s'auto-affecte, la formation de [cette extériorité] relève toujours de l'affectivité, l'affectivité de la représentation est celle du pouvoir qui la forme - ou qui ne la forme pas. Ce dernier cas est celui du refoulement dont l'énigme se dévoile à nos yeux. ${ }^{42}$

Il est possible de connaître la représentation avant qu'elle ne soit parce que «ce n'est pas elle qui peut nous instruire sur elle-même et nous inviter à l'écarter, ce n’est pas son contenu représentatif, mais son affectivité :

${ }^{41}$ Michel Henry, GP, p. 233.

${ }^{42}$ Ibid., p. 233-234. 
Si la représentation ne déplie pas son contenu devant l'esprit, ce ne peut être dès lors qu'en fonction de son affectivité : c'est le déplaisir, c'est la souffrance, c'est à la limite la terreur liée à cette représentation, non ce qu'elle représente, qui motive sa mise à l'écart de la conscience, sa non-venue devant le regard de celleci, sa non émergence dans le Dehors de l'extériorité. Mais l'affect de la représentation était déjà là et c'est lui en réalité, et lui seul, qui a décidé d'exclure le contenu représentatif, de lui faire subir le refoulement. ${ }^{43}$

Ce n'est donc pas le contenu de la représentation qui va être connu avant qu'elle ne soit exclue. C'est une force étrangère à la représentation, comme l'affirme Schopenhauer. Mais, ajoute Henry, la force qui forme ou ne forme pas la représentation n’est pas soustraite à la phénoménalité, elle n’est pas réellement inconsciente. Elle est l'« Archi-phénoménalité " ${ }^{44}$ de l'Affect. Celle-ci est caractérisée par Henry comme un «savoir sans représentation, savoir avant la représentation, savoir secret de toute représentation, qui sait déjà ce qu'elle va représenter, qui lui permet de le faire ou le lui interdit " ${ }^{45}$. Ce savoir sous-jacent, opérant et qui peut être refoulant, est celui de la vie en son essence phénoménologique propre. Il est l'affect consubstantiel à cette phénoménalité non ek-statique, celui qui ne saurait en être séparé en tant qu'elle est issue de la dynamique d'un sujet incarné : « En sorte que [...] placé au cœur de l'inconscient, l'affect détermine celui-ci comme phénoménologique en son essence et en son fond. ${ }^{46}$ Mais l'affect lui-même, dans la lecture d'Henry, présenté comme source de savoir et lieu d'une efficacité formelle, ne devient jamais inconscient. Henry prétend, par cette lecture, reprendre le constat de Freud. Si parfois on parle d'affects refoulés, « le refoulement ne signifie donc ici aucune disparition de l'affect ni par conséquent de la phénoménalité qui lui appartient par principe, mais seulement sa modalisation en un autre affect ${ }^{47}$; tandis que, dans le refoulement, la représentation liée à l'affect n'est pas formée, " l'affect lui-même n'est pas supprimé mais modifié qualitativement, devenant telle ou telle tonalité. Quand celles-ci à leur tour sont interdites, refoulées, c'est dans l'angoisse qu'elles se changent en même temps que l'affect primitif ${ }^{48}$. Cette affectivité indépassable (en deçà de laquelle il n'y a rien que l'activité incessante du corps vivant et l'ensemble de ses pouvoirs et de ses forces) révélerait une subjectivité incarnée qui, par cela même qu'elle n'est pas à son origine, trouve son activité enracinée dans une passibilité de fond, celle de la Vie, avant le monde, dont les lois se confondent avec celle du désir.

\footnotetext{
${ }^{43}$ Michel Henry, « La question du refoulement », dans PV-II, p. 144.

${ }^{44}$ Ibid.

${ }^{45}$ Michel Henry, GP, p. 234.

${ }^{46}$ Ibid., p. 10.

${ }^{47}$ Ibid., p. 370

${ }^{48}$ Ibid.
} 
C'est donc, nous dit Henry à la suite de Schopenhauer et de Nietzsche, qu'il faut situer ce qu'il y a de mieux dans les textes de Freud. Les meilleurs seraient justement ceux où le primat de l'affectivité affleure, où se fait jour «cette subordination de l'intellect à la vie ». La théorie et la thérapie psychanalytiques mettraient d'abord en évidence «cette subordination de la pensée représentative perceptions, images, souvenirs, productions oniriques et symboliques, esthétiques et religieuses, etc. - et de tout ce qui se montre en elle, à un pouvoir d'un autre ordre ${ }^{49}$. Ce sont aussi ces textes qui présentent la situation clinique comme étant ancrée dans la dynamique affective du transfert, qui en est le principal moteur. Ils montrent que la représentation par l'analysé de sa propre situation, de ses conflits et de leur histoire, ne sert à rien aussi longtemps qu'une modification affective n'accompagne cette conscience, " c'est-à-dire [aussi longtemps] qu'une modification de la vie, n'est pas intervenue $"^{50}$. L'acceptation intellectuelle du sens n'est rien sans une transformation affective qui ouvre de nouvelles possibilités de vie. Cette subordination s'étend à la détermination de la valeur de vérité des interprétations et des constructions, qui sera dépendante, elle aussi, de la teneur affective des rapports établis dans la communauté formée par l'analyste et l'analysant.

\section{Pourquoi l'inconscient est-il l'un des noms de la vie ?}

Compte tenu de cette subordination de la pensée et de la représentation à l'affectivité, dégagée à partir de sa compréhension du refoulement, il est possible de mieux comprendre le statut que Michel Henry assigne à l'inconscient. Mais y a-t-il encore un sens quelconque à parler d'inconscient si nous savons que le principe qui y est à l'œuvre est l'affectivité, et que celle-ci est toujours présente, même dans les rêves? Quelle signification ce terme peut-il avoir après la critique de Michel Henry ? L'auteur de la Généalogie de la Psychanalyse décèle sous la plume de Freud une double caractérisation du terme d'inconscient, selon le rapport que celuici entretient avec un savoir de la vie qui précèderait toute objectivité et qui serait celui d'une subjectivité absolue où toute représentation de soi, des autres ou du monde trouve son origine. C'est ce savoir de la vie, en fonction duquel est inhibée ou formée une représentation, qui servirait de critère pour la distinction de la double signification du concept d'inconscient. Il « voulait [...] dire deux choses totalement différentes selon qu'on se référait à l'obscurité en laquelle sombre inévitablement tout contenu conscientiel dès qu'il quitte le présent de l'intuition ou de l'évidence pour n'être plus qu'une représentation virtuelle, ou au contraire à la vie elle-même en tant qu'elle se dérobe par principe à la lumière de l'ek-stase » ${ }^{51}$. Selon Henry,

${ }^{49}$ Ibid., p. 10.

${ }^{50}$ Ibid., p. 385.

${ }^{51}$ Ibid., p. 388. 
cette double référence se produit constamment chez Freud. Une grande partie de l'avant-dernier chapitre de la Généalogie de la Psychanalyse est consacré à montrer comment la simple latence des contenus représentatifs cède peu à peu la place à « une considération plus profonde qui thématise l'inconscient dans sa connexion à l'essence de la vie et au mode primitif de son accomplissement immanent : l'action, la force, la pulsion, l'Énergie " ${ }^{52}$. Si la première caractérisation de l'inconscient succombe à la critique d'une phénoménologie radicale, l'autre, en revanche, se voit éclairée par celle-ci et se voit rangée parmi les formes que prennent l'immanence de la subjectivité vivante et son savoir immémorial et charnel.

Le premier cas est celui de l'inconscient de la "représentéité »; il se propose comme l'ultime illusion d'une métaphysique de la représentation et comme le revers nécessaire et indissociable du cogito. Dans la mesure où il repose sur la virtualité de la plupart de nos représentations, il présuppose la croyance en l'existence réelle de celles-ci sous la forme de contenus discrets juxtaposés dans un lieu inventé à la seule fin de les recueillir en lui. Mais, dans l'inconscient, les représentants refoulés, pour être inconscients, gardent paradoxalement l'essence «extatique de la phénoménalité - en l'absence de celle-ci toutefois, en l'absence de l'effectivité phénoménologique que l'ek-stase constitue en elle-même et par elle-même " ${ }^{53}$. Il s'agit d'un inconscient réaliste, effet de la latence des représentations virtuelles, qui aboutit à «l'hypostase de la structure ontologique des représentations, de l'extatique-horizontal en tant que tel ». Il n’y a plus place pour une phénoménologie de la vie dès lors que l'on a admis que la représentation refoulée - la représentation dont la réalité formelle [qui est affective] empêche la réalité objective - n'est pas formée ; n'est pas non plus dans quelque topos de la vie psychique où elle subsisterait.

Ce qui subsiste [en revanche] c'est une tonalité, se profilant dans la première sphère de l'être, comme un accident, ou s'y déployant à titre d'habitus. Point n'est donc besoin pour rendre compte du refoulement de la mythologie des topiques ni de leurs personnages plus ou moins grossièrement empruntés au monde de la représentation, le savoir de la vie y suffit. ${ }^{54}$

Cette inutilité du refoulement s'étend aussi à l'inconscient ainsi entendu, qui en est inséparable. En revanche, l'autre aspect de ce que Freud nomme inconscient a la plus grande importance, en tant qu'il rend compte de l'« en-deçà du monde où s'édifie intérieurement l'essence de tout pouvoir " ${ }^{55}$. « En-deçà du monde " veut dire, chez Henry, «dans l'invisible, dans l'immanence radicale de la subjectivité absolue. Ainsi est-on reconduit chez Freud lui-même d'une première définition

\footnotetext{
${ }^{52}$ Ibid.

${ }^{53}$ Ibid., p. 389.

${ }^{54}$ Ibid., p. 234.

${ }^{55}$ Ibid., p. 392.
} 
superficielle de l'inconscient à une autre, plus significative » qui se confond avec l'immanence radicale du corps, de son activité, de ses pouvoirs et de la passibilité de la chair impressionelle. Et c'est bien de cette façon que Freud touche à l'essentiel et à ce Commencement perdu par une métaphysique de la représentation qui a cherché la Vie dans l'ek-stase où elle ne se donne jamais. Cet « inconscient efficient $»^{56}$ est la source irreprésentable de la représentation, le revers subjectif, inobjectivable de l'objectité, l'épreuve de soi d'une vie s'exprimant par l'incessant des forces où se forme la teneur affective et sémantique des rapports entre sujets et de leurs praxis. Là se trouve, également, la source de ce qui est donné comme monde à l'intentionnalité de la conscience, ainsi que la source des comportements humains. Cet inconscient, qui n'ek-siste donc pas, est - sous la plume de Henry - «le nom de la vie $»^{57}$.

\section{La connaissance psychanalytique est-elle une forme de désespoir?}

En raison de ces deux formes que peut prendre l'inconscient et de sa signification, la psychanalyse serait traversée par un mouvement contradictoire. Par le biais de son analyse phénoménologique, Henry repère chez Freud à la fois les restes de la métaphysique occidentale centrée sur le cogito - dont il serait un " épigone » ${ }^{58}$ et un « héritier tardif $~^{59}$ - mais aussi un dépassement de cette même métaphysique rendu possible par la Volonté schopenhauerienne et par la Vie nietzschéenne. D’une part, la psychanalyse redécouvre l'importance décisive de la vie et de son pathos dont l'essence est pulsionnelle. En tant que telle, la découverte freudienne constituerait le dernier terme de la reconnaissance d'une subjectivité où se trouve ce même savoir, à une époque où prime une idéologie de l'objectivité. Mais d'autre part, traversée par une visée scientifique, et alors même qu'elle découvre la source du savoir là où s'enracine l'activité subjective incarnée, elle souhaiterait s'en débarrasser et agirait à sa façon le désespoir qui consiste à vouloir (s') arracher une partie de soi-même en séparant l'activité propre à la pensée représentative (où se situent la clinique dès lors qu'elle met l'accent sur l'aspect verbal et sur le retour à la conscience) de la passibilité subjective. Le travail de la cure aurait en partie pour tâche de placer la seconde sous les logiques de la première en reconduisant au plus proche de zéro l'excitation à l'origine de toute l'activité pulsionnelle non inféodée à l'ordre de la signification consciente et verbalisable.

Le constat métaphysique se double alors d'une critique de la connaissance psychanalytique. Cette critique concerne avant tout le privilège accordé à la

\footnotetext{
${ }^{56}$ Ibid.

${ }^{57}$ Ibid., p. 348.

${ }^{58}$ Ibid., p. 8.

${ }^{59}$ Ibid., p. 5.
} 
représentation, qu'elle soit visuelle ou symbolique, au détriment du pouvoir irreprésentable de pâtir, avec lequel se confond la subjectivité. La critique s'étend donc à une métapsychologie fixée une fois pour toutes, et qui servirait de référence explicative à toute occurrence psychique, mais elle concerne aussi chaque réduction de la subjectivité vivante - praxis in-objectivable du savoir - à des modèles et à des hypothèses issus des sciences humaines, notamment de la linguistique, ou des sciences naturelles. D’un côté, c'est l' « École de Paris » de J. Lacan qui est visée, et Henry déclare que «la contamination ou la dénaturation de la psychanalyse par la linguistique et l'ensemble des disciplines qui lui sont aujourd'hui associées est [...] possible [dès] que l'on va pouvoir déclarer sans rire que la structure de l'inconscient est celle d'un langage ${ }^{60}$, mais c'est aussi, d'un autre côté, la tendance initiale de la psychanalyse, l'inspiration neurophysiologique de départ qui jamais ne quittera Freud, que l'on retrouve dans sa théorie des pulsions et en raison de laquelle (et même lorsqu'il introduit l'Eros) - il semble exprimer un pessimisme ou en tout cas une méfiance fondamentale à l'égard de la vie, de son excitation sans cesse renaissante et des sources immaîtrisables du désir et de l'affectivité. Par le dessein de connaissance qui la guide dès l'origine, la psychanalyse freudienne participerait partiellement de l'effort pour se débarrasser de la vie en tant qu'immédiateté autoaffective, antérieure au monde fait d'horizons et de pro-jets. Le freudisme, écrit Michel Henry, « est le dernier jalon de cette histoire qui, contestant la définition de l'homme par la pensée, découvre au plus profond de lui la vie. Mais le freudisme n’a pris en compte la vie que pour la liquider ${ }^{61}$. Le mouvement que l'on trouve à la fois dans le désespoir, dans le refoulement et dans la psychanalyse, serait celui d'une négation originaire inhérente à la constitution du sujet de la connaissance, écartant et maintenant à distance - oubliant et occultant - une passibilité de fond et un savoir qui s'y révèlerait à son insu. Dans ce mouvement par lequel le sujet s'efforcerait de s'arracher une partie de soi-même, se révèlerait négativement à la fois le statut primordial de l'affectivité comme auto-révélation et comme savoir anté-prédicatif d'une activité invisible, ainsi que son enracinement dans l'immanence d'une vie dont il n'est pas à l'origine, source absolue de chacun des pouvoirs et de son incessante activité.

\footnotetext{
${ }^{60}$ Ibid., p. 356. Henry semble, néanmoins, revenir sur son propos et le rendre moins tranché en affirmant, quelques années après la parution de la Généalogie de la Psychanalyse, dans l'article intitulé « Phénoménologie et psychanalyse », que sa lecture de la psychanalyse n’avait pas pour but d'écarter les interprétations qui avaient prévalu jusqu'à ce moment-là, notamment "la lecture langagière qui s'était imposée en France durant les [...] décennies [précédentes]. Il ne s’agi[ssait] pas de sous-estimer des phénomènes aussi importants que le langage non plus que le rôle qu'il joue à l'évidence dans la cure analytique " (Michel Henry, "Phénoménologie et psychanalyse », op. cit., p. 105). Le langage doit être reconnu comme n'étant que le « logos du monde » ne devant pas être pris de manière exclusive, sans quoi on occulte ce qui se tient derrière lui. Le langage est moins essentiel que l'affect et son dynamisme, qui « constituent proprement la réalité ontologique dont le langage n'est qu'une formulation après-coup » (Ibid., p. 105).

${ }^{61}$ Michel Henry, GP, p. 382.
} 
Un texte de Freud fait écho à cette remarque de Michel Henry. Dans Die Verneinung $^{62}$, la forme négative du jugement, qui signale le refoulement, exprime, au niveau de la représentation verbale, le mouvement de refus et l'effort de mise à l'écart des significations non admises au niveau conscient. Cette forme pulsionnelle de rejet, portant sur des représentants psychiques, reprend une expulsion initiale et une négation de départ par lesquelles s’instaure la séparation entre le dehors et le dedans, le moi et le monde. C’est une négation originaire - qui a scindé le narcissisme primaire, le séparant entre un dehors composé de ce qui est mauvais et un dedans où est admis ce qui est bon - qui instaure un écart qui rend possible la position d'un ob-jet (Gegenstand) se maintenant en vis-à-vis. La chose mauvaise de départ, mise au dehors, mise en tant que dehors, s’avèrerait génétiquement être, dans la lecture d'Henry, une part de cette Vie absolue oubliée où prendrait des racines la séparation entre d'une part, le moi pensant et connaissant et d'autre part, le monde. Les deux parts seraient mues par cela même qui a été rejeté par une subjectivité pour que celle-ci s'instaure. On comprend alors pourquoi Henry, alors qu'il ne fait pas référence à ce texte de Freud, peut émettre cette hypothèse selon laquelle une connaissance représentative (en raison de sa médiation symbolique) comme forme d'accès à soi serait en dernière analyse l'expression d'un effort de scission d'avec la dimension pathique, résultat d'une pulsion destructrice initiale et souhaitant évacuer les indices de la passibilité originaire propre au Fond de la Vie. Cette tentative de la vie de se détruire elle-même, écrit Henry, « en se séparant de soi, de se détruire ou de se connaître, ne détruit pas plus la vie qu'elle ne la connaît, n’est qu'un mode nouveau de cette vie, une forme de désespoir " ${ }^{63}$. L'effort de la connaissance, en cela qu'il ne se situerait qu'au niveau de la symbolisation corrélative à l'écart objectivant et négligerait la dimension pathique, agirait " thétiquement » ce désespoir et participerait à ce mouvement tendant vers la diminution des pouvoirs de la vie présent dans le refoulement.

En mettant l'accent sur la médiation symbolique, la psychanalyse ouvrirait la porte à l'infinité de la tâche herméneutique, tout en barrant l'accès à cette dimension muette où le sujet se donne à soi dans la simplicité de l'affection pathique et pulsionnelle par laquelle s’apparaissent les membres de toute communauté vivante. Par son insistance sur le devenir conscient du refoulé par l'interprétation et la remémoration, la clinique psychanalytique semble s'avérer incapable de révéler la vie inobjectivable en deçà de la conscience et de la représentation. Alors qu'elle est l'héritière d'un courant de la métaphysique qui a remis en question le primat du cogito, la psychanalyse aboutirait néanmoins à «l'établissement $\mathrm{d}[\mathrm{u}]$ règne sans partage [de la représentation] » ${ }^{64}$ qui asservit l'affectivité à la médiation symbolique

\footnotetext{
${ }^{62}$ Sigmund Freud, «La négation », trad. par J. Laplanche, dans Résultats, idées, problèmes II, Paris, PUF (« Bibliothèque de Psychanalyse »), [1925] 1985, p. 135-140.

${ }^{63}$ Michel Henry, EM, p. 855.

${ }^{64}$ Michel Henry, GP, p. 194.
} 
par laquelle se maintient la dimension intentionnelle. Ce faisant, elle perd le Commencement et écarte de l'essence de la subjectivité absolue comme autoaffection. Les pensées de la médiation utilisent le détour pour savoir ce que nous sommes. Soit notre être véritable ne se constitue que médiatement, par le biais d'un rapport aux autres, soit la connaissance que nous pouvons en avoir n'est jamais ellemême que médiate, passant nécessairement par les symboles et les institutions d'une culture. Au lieu de mener à la connaissance de soi, elle renforcerait l'effort pour se débarrasser de soi qui est le propre du désespoir. Cela concerne au plus haut point la clinique mise au point par Freud et vise indirectement la lecture qui en est faite par Ricœur ${ }^{65}$, puisque

pour surprendre le secret de notre être, la psychanalyse a abondé dans ce sens. La pulsion ne se manifeste que par ses «actes», par l'ensemble éclaté des comportements inaperçus du sujet, de ses représentations, de ses affects : autant d'indices offerts à une lecture herméneutique. Il faut donc se mouvoir à travers une forêt de symboles pour tenter de repérer les grandes lignes le long desquelles la pulsion a tenté de se décharger - et la vie de se débarrasser de soi. ${ }^{66}$

\footnotetext{
${ }^{65}$ On peut lire à ce propos les lettres de Michel Henry à Paul Ricœur, dont «l'admirable analyse du grand essai de Freud sur l'inconscient [a] guid[é] » la réflexion de Henry (RMH 1, p. 16). Cf. aussi Michel Henry, " Ricœur et Freud : entre psychanalyse et phénoménologie », dans PV-II, p. 163183.

${ }^{66}$ Michel Henry, GP, p. 397.
} 
